\title{
Independence of memory for categorically different colors and shapes
}

\author{
D. LEO STEFURAK and ROBERT M. BOYNTON \\ University of California, San Diego, La Jolla, California
}

\begin{abstract}
Subjects carried out a difficult mental arithmetic task while viewing five colored forms. After a variable delay period, they indicated whether a randomly selected test item had a new color or shape aspect. If the test item appeared old in both aspects, then the subject indicated whether or not color and shape were conjoined as at inspection (Experiment 1). Correct mental arithmetic and recognition responses qualified the subject for monetary rewards. In Experiment 2, subjects were attentionally primed to the color or shape dimension and were asked to indicate if the test item was old or new along that dimension. The nonattended dimension acted either as a recognition facilitator or as a distractor. The stimulus features were categorically different within dimensions, and naming was inhibited by resource competition from the arithmetic task. Under these conditions, complete independence of the visually mediated memory for form and color was observed. In Experiment 1, memory for color-shape conjunctions was totally absent, and in Experiment 2, neither facilitation nor inhibition was observed as the nonattended dimension was varied.
\end{abstract}

A dual-aspect stimulus is an item that has features selected from two dimensions, such as color and shape or size and brightness. Populations of such dual-aspect stimuli have an additional parameter: featural relationship. Conjunctions of features exist in object space as a proximity relationship among features; if such conjunctions are remembered, then there must be a representation in cognitive space as well. The current tide of studies on percept construction runs against models that lock feature conjunctions at any early stage of representation. Research by Garner (1977) and Treisman and Schmidt (1982) into the stimulus integration properties of human perception have led to a model suggesting that features from each dimension of a stimulus are separately encoded. A feature integration stage follows in which conjunctions are explicitly represented both within and between dimensions. Treisman (1977) notes various ways in which these conjunctions could be represented and held in a working memory for use in a later recognition task, and provides evidence that, for brief simuli, illusory conjunctions may occur between features of discrete stimulus items. For example, a red circle might be reported as one member of a set of stimulus items that included a red square and a green circle among other items neither red nor circular.

According to current models concerned with the perception and memory of a collection of dual-aspect stimuli, the

The authors would like to acknowledge the assistance of Jeffrey Miller in the design of Experiment 1. His insight and comments were invaluable in achieving a productive and interpretable design. We would also like to thank our co-experimenters, Ellen Ruskin and Conrad Olson, without whose diligence this work could not have been accomplished. This work was supported by Grant EY-01451 from the National Eye Institute and by a Kollmorgen Foundation fellowship to the first author. Requests for reprints may be addressed to D. Leo Stefurak or Robert M. Boynton at the Department of Psychology, C-009, University of California at San Diego, La Jolla, CA 92093. presentation of a test probe is followed by a sequence of perceptual processes (Santa, 1977). An on-line or memory search occurs for a given feature first along one dimension and then along the other dimension. Only after this search is completed will a check be made of the possibility of a conjunction match between the representation of the probe and that of the original items in the stimulus set. One of Treisman's main findings is that focal attention is necessary for the melding of features into a conjoint entity. The overloading of attentional resources produces conjunction errors and real misperceptions of dual-aspect stimuli, resulting in the perception of illusory objects.

With these points in mind, the current study examines, for two-dimensional stimuli, the role of verbal coding in recognition memory. Members of a set of solidly colored and equally sized objects are visually discriminable only if they differ in shape or color, or both. Here we examine short-term memory for sets of stimuli that differ categorically in these two ways, as defined by the fact that the colors and shapes chosen for study are overlearned, never confused, and reliably named.

Memory for items discriminable by color and shape is usually mediated by verbal labels to such an extent that some have suggested that no truly sensory memory exists. For example, Schiano and Watkins (1981) concluded that "there is a substantial functional equivalence between shortterm memory for readily nameable pictures and for words and ... this equivalence may be thought of as ... mediation by a common, 'speech-like' code"' (p. 110). To test (and reject) the hypothesis that verbal labels necessarily mediate memory for form and color in a situation in which names ordinarily would be used, we obtained data using a competing task designed to greatly inhibit verbal encoding, and compared these results with those from control conditions in which verbalization was not inhibited. Results from one experiment suggested that both colors and forms 
can be rather well remembered without verbalization; however, without the use of a verbal code, there is no memory whatever of their conjunction. A second experiment showed that, without verbalization, discrete memory for form or color is not influenced by the nature of, or changes along, the other dimension.

As an isolated variable, color has been investigated relative to large, categorically different and verbally codable colors by Bartleson (1960), Herring and Bryden (1970), and Heider and Oliver (1972). There appear to be exactly 11 categorically different colors to which distinct names are universally attached. Ratliff (1976) provides convincing reasons for believing that the underlying physiology of the human visual system is responsible for the remarkable consensus about these color terms. Categorically different shapes also elicit name tags in quite a natural way, and at least some of them may have a physiological basis that partially transcends the arbitrary learning experience of a particular organism. As in the case of color, opinions differ concerning whether shapes can have a purely sensory representation (see Paivio, 1969, for a review).

Suppose that members of a group of objects differing in shape must be remembered and subsequently recognized. If each of these objects were given a unique color, it would seem reasonable to conclude that a given shape would be easier to remember with this redundant conjoint color than if all of the shapes were the same color. Similarly, one might expect that colors would be easier to remember if each color were uniquely associated with different object shapes, rather than if presented abstractly or attached to indistinguishable amorphous blobs. Our results contradict these commonsense expectations, suggesting that, if the use of verbal labels is inhibited, memories for form and color behave independently and seem to rely upon noninteracting sensory processes.

\section{EXPERIMENT 1}

\section{Method}

Stimuli. A single stimulus item consisted of one of 10 animal silhouettes, each glued to a $7.6 \times 7.6 \mathrm{~cm}$ light-gray background. The animal forms were scaled and equated for area, and are shown in Figure 1. Each animal was made in 10 solid colors, and 100 unique dual-aspect stimuli formed the stimulus pool. The physical specifications and common names of the colors are given in Figure 2 .

Ten colors were selected that correspond to the basic categories (excluding gray) described by Berlin and Kay (1969). Various construction papers, specified in Figure 2, were selected that seemed to be good focal examples of these colors, and pretesting was done with 8 subjects, not used in the main experiment, who were asked to identify the stimuli using single color names. Animal shapes were selected on the basis of pretesting from a larger population of 15 candidates.

Later, in preliminary sessions, the 4 subjects of the first experiment and the 8 subjects of the second experiment were shown samples of the eight colors and eight animals under experimental viewing conditions. With a few exceptions, ${ }^{1}$ they were consistent in naming both colors and animals with single words.

Apparatus. The subject looked through a round 2.5-cm-diam hole into a plywood box that measured $60 \mathrm{~cm}$ on a side and was painted flat white inside. Stimulus items were placed by hand on the bot-
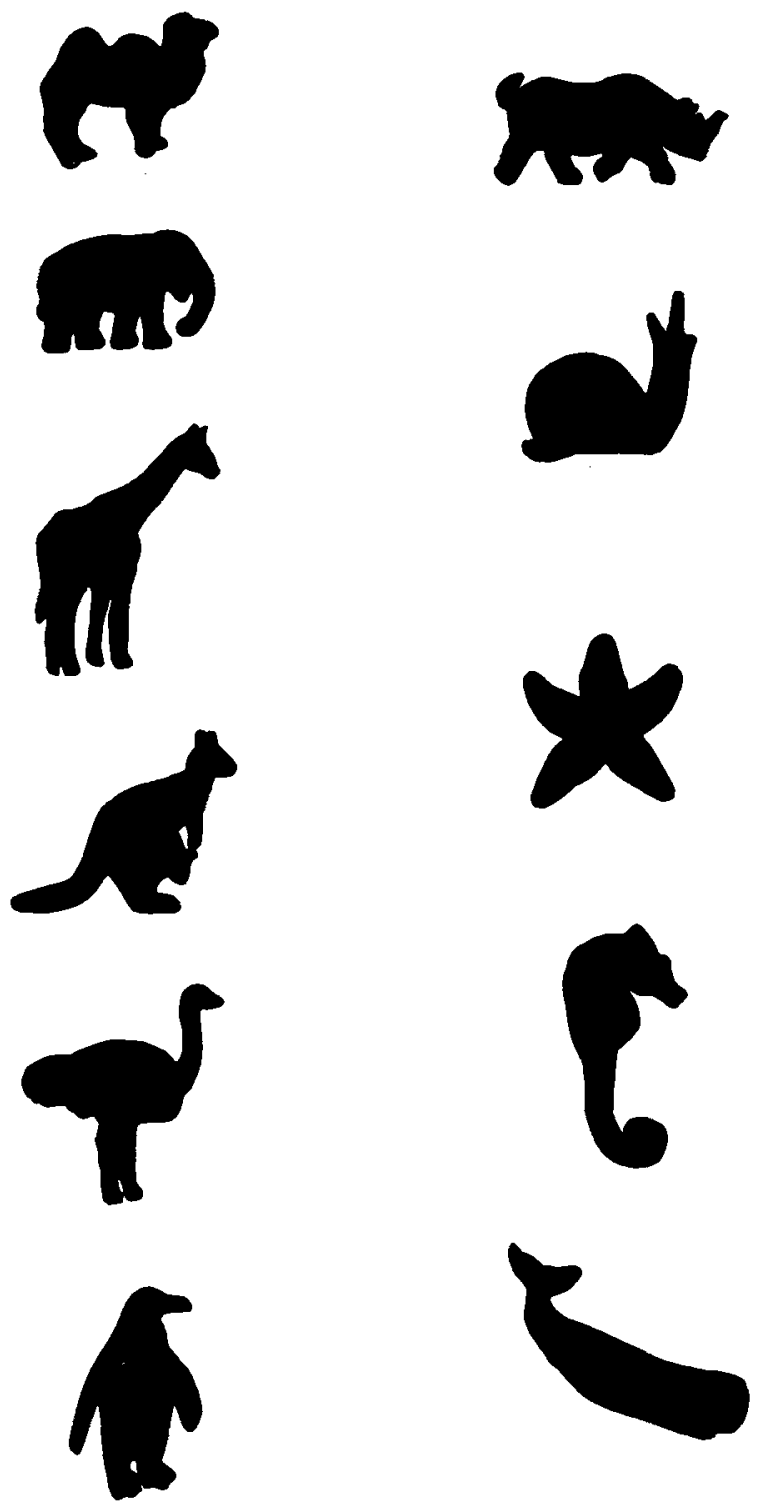

Figure 1. Animal shapes: The 10 equal-area animal silhouettes used throughout both experiments. With the minor exceptions given in the footnote, these were called camel, elephant, giraffe, kangaroo, ostrich, penguin, rhinoceros, snail, starfish, seahorse, and whale. (The starfish shape was used only in Experiment 2.)

tom of the box and were seen reflected by a diagonally placed mirror as if vertically oriented on a back wall of the box at a distance of $66 \mathrm{~cm}$. A Uniblitz shutter, controlled by a digital timer, was positioned $6 \mathrm{~cm}$ in front of the observer's right eye. Two 60 -W incandescent bulbs, operated at line voltage (about $120 \mathrm{~V}$, with a color temperature of $2,650 \mathrm{~K}$ ) and mounted in recesses on each side of the device, indirectly illuminated the stimulus items. A contoured port provided reference for head position. Except for control conditions, an audio cassette tape player, whose loudness was adjustable by the observer, ran continuously throughout each experimental trial.

The subject, who was required to respond within $4 \mathrm{sec}$ after onset of the test stimulus, signaled his or her response by pressing a button. Only once during the 3,460 trials of the two experiments combined did a subject fail to accomplish this.

A digital counter, activated by the experimenter, was displayed above the viewing port. Each increment of the counter signified 


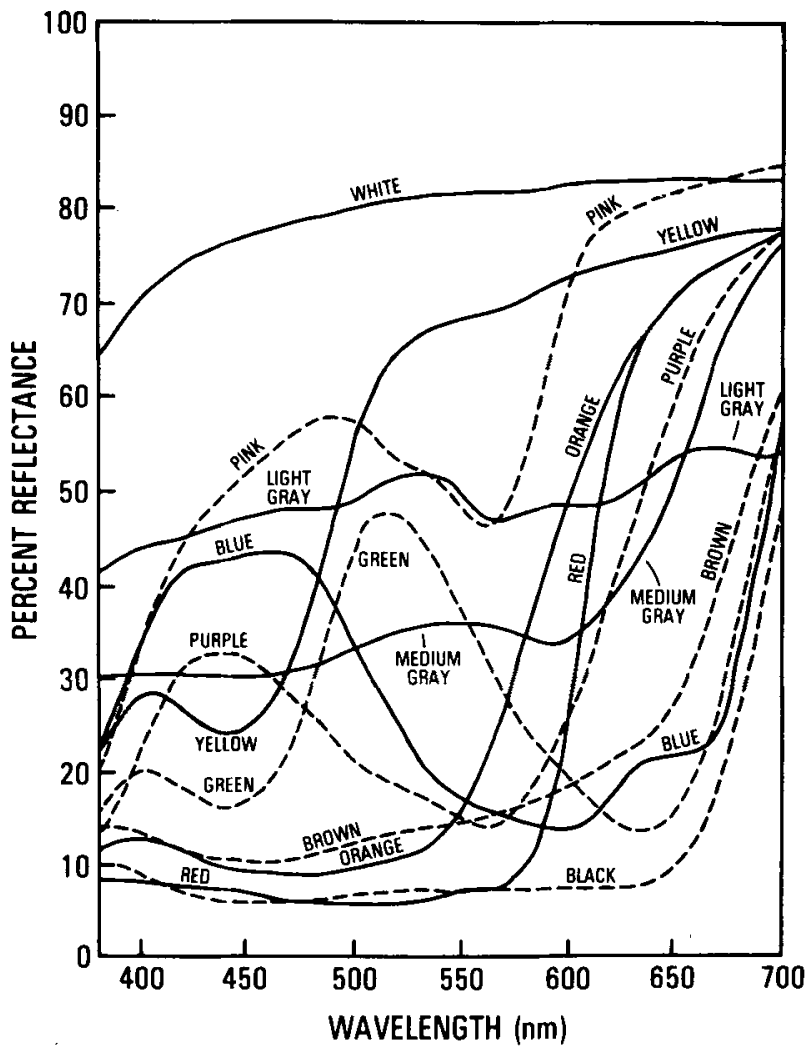

Figure 2. Spectral reflectance curves: The percent diffuse reflectance versus wavelength of the colored construction papers used as stimulus materials, as measured by the Hemmendinger Color Laboratory, Belvidere, NJ.

a reward of $\$ 0.10$. The experimenter also had access to a bell used to signal a bonus reward of $\$ 1.00$.

Procedure on a typical trial. A trial began with the onset of a voice from the tape player saying "ready," followed by a voiced integer chosen randomly between 1 and 9 inclusive. This marked the beginning of a mental arithmetic problem that would continue throughout the experimental trial. After a $2-\sec$ delay, a statement such as "plus 3" or "minus 6" was heard every 2 sec. The integer to be added or subtracted by the subject to update the cumulative total was chosen randomly between 1 and 8 with the restriction that no correct answer could fall outside the range of 1 to 9 inclusive. Except when not permitted by the latter constraint, there was an equal probability that addition or subtraction would be required. The subject was instructed that no reward could be obtained unless, after verbalizing his response to each stage of the arithmetic problem, he also produced a correct final answer at the end of the trial.

After three or four answers to the arithmetic problem had been given, the shutter opened to expose a set of five colored animal shapes for $5 \mathrm{sec}$. The array of five animal shapes subtended $13^{\circ}$ of visual angle vertically and $9^{\circ}$ horizontally, arranged in an $\times$ like array. After the shutter closed, the experimenter removed the five-item tray and substituted a single test item. At the conclusion of the delay period, the shutter opened for a second time, exposing the test item until the subject responded by pressing a button while continuing to verbalize answers to the arithmetic problem.

Three response buttons were used in the first experiment. One of these was used to signal the judgment that either a new color or shape (or both) was seen at test. A second button indicated that the test item was judged to have been identical to one of those present in the inspection set: an old color, old shape, and old conjunction.
The third button was used to indicate that both the color and shape had been present at inspection, but not conjoined: an old color, old shape, but new conjunction. The arithmetic problem continued until well after the buttonpress. After the final answer to the arithmetic problem had been given by the subject, the voice from the tape player stated the correct final arithmetic sum. No verbal feedback was given for buttonpress responses; in some cases, according to a schedule (see below), a reward followed. The next trial began after an interval of about $55 \mathrm{sec}$.

Experimental design. The within-sessions variables were:

1. Delay between inspection and test. With the constraint that each delay would occur either 22 or 23 times in a session of 45 trials, delay intervals of 3 and $15 \mathrm{sec}$ were varied randomly from trial to trial. Delay was counterbalanced across sessions and subjects for the 22 or 23 trials in each delay interval.

2. Inspection stimuli. From a set of 100 possible color $\times$ animal stimulus items, 5 were chosen randomly on each trial and positioned according to the order of their selection. The choice of the 5 stimulus items was constrained to prevent repetition of color or shape within the inspection set. Half of the shapes and half of the colors were thereby represented.

3. Test stimuli. One item from the set of 100 was chosen randomly for presentation on each trial with the constraint that each of the three stimulus-response conjunction categories (defined below) be represented 15 times in a session.

4. Procedure. At the start of each session, three unrewarded practice trials reminded the subject of the response categories and button use. Forty-five experimental trials were then run with a 5-min break approximately midway.

Subjects. Undergraduate subjects were solicited by means of a posted notice. The vision of all subjects was normal, with optical correction if needed. All passed the Farnsworth-Munsell 100 Hue Test (red-green tray only) with a score of less than 20 . All 4 original subjects completed the experiment.

Sessions and Conditions. The subjects participated in six sessions. The initial session for each subject, which took about $2 \mathrm{~h}$ to complete, included pretesting (color naming and the FarnsworthMunsell test), instructions read by the experimenter, and 45 practice trials with rewards and mental arithmetic. Each of the next four experimental sessions took about $90 \mathrm{~min}$ to complete and included only trials for which the subject performed mental arithmetic. The sixth session was used as a control. It differed from the preceding four sessions in that the subjects were not required to perform the mental arithmetic. After data collection was complete, a final $30 \mathrm{~min}$ was used for debriefing and discussion of the experiment.

For the experimental conditions, the subject was told that each correct-response category was represented equally among the 45 trials of a session. Each subject therefore received 60 trials over the course of four experimental sessions in each of the three recognition memory conditions. Each condition corresponded to one of the response categories: new structural feature, old conjunction, new conjunction (see below).

Recognition task. The three-alternative forced-choice paradigm was used to determine which test features were recognized from the inspection set. One alternative indicated a new structural feature: color, shape, or both in the test item were judged to be novel (not seen among the five inspection items). The remaining two alternatives were appropriate when both the color and shape of the test item were believed to be "old" ones (i.e., present somewhere among the 10 discrete structural features of the five inspection items). The old color and old shape could be conjoined in the test item as they were for one of the inspection items; this was the second possible response. Or the old color and old shape could be conjoined differently in the test item than at inspection; this was the third possible response.

Rewards. Eligibility for a reward depended upon the joint occurrence of a correct final sum on the arithmetic task, and a correct response on the recognition task. If these conditions were met, 104 rewards were given with 0.67 probability. On a given trial, 
there was 1 chance in 13 that a subject would also be eligible for the $\$ 1$ bonus reward, with the exception that one such trial was spotted randomly within sessions which otherwise would not have had one. Verbal knowledge of results within sessions was never given, nor were subjects told whether a forthcoming trial would be a reward trial or what the delay interval would be.

At the end of a session, the observers were informed about how many bonus trials had occurred during that session and how many of these they had missed. Then the subject was given a voucher redeemable for $\$ 3.00$, a payment not contingent on rewards. The winnings were accumulated across sessions and paid out in the form of a redeemable chit at the end of the sixth session. Winnings would have been forfeited had the six sessions not been completed. The span of six sessions lasted no more than 3 weeks for any observer, and all sessions for a given observer occurred on separate days.

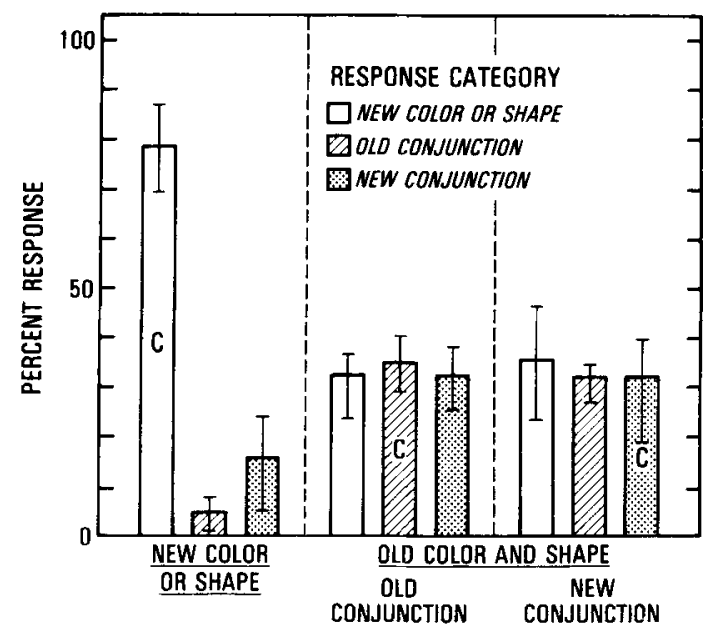

STIMULUS CONDITION

Figure 3. Main results from Experiment 1: Response distributions for conditions using the arithmetic distractor task. Error bars give the range of intersubject variability.

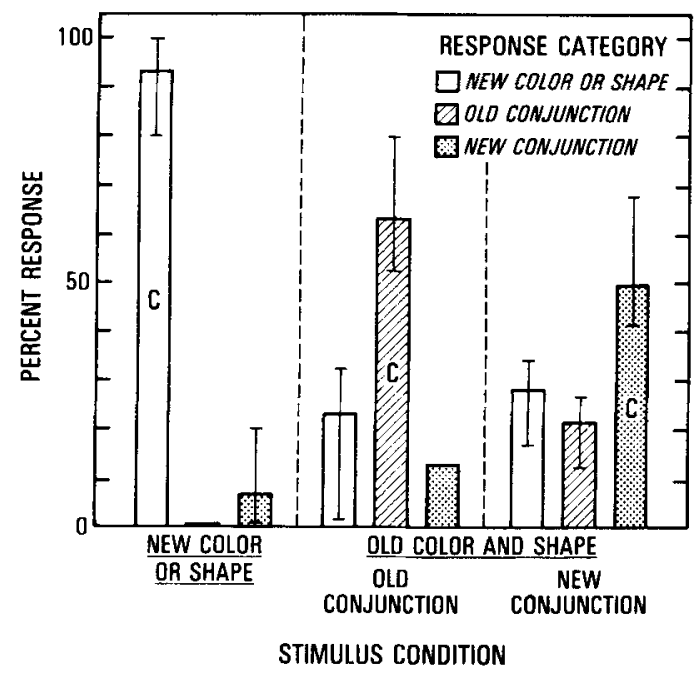

Figure 4. Control results from Experiment 1: Response distributions for the condition in which the arithmetic distractor task was absent. Error bars give the range of intersubject variability.
Table 1

Results of Statistical Tests for Experiment 1

\begin{tabular}{lll}
\hline Recognition performance between the 3 conditions & $d f$ & $F$ \\
Experimental trials with counting & $(2,9)$ & $52.22 \dagger$
\end{tabular}

Control trials without counting $\quad(2,9) \quad 15.35$ †

Response performance between the 3 response categories

Experimental trials with counting (see Figure 3)

New color or shape stimulus condition

Old conjunction stimulus condition $\quad(2,9)$

New conjunction stimulus condition

$153.99+$

Control trials without counting (see Figure 4)

New color or shape stimulus condition

$(2,9) \quad<1.00$

$(2,9) \quad 183.00 \dagger$

Old conjunction stimulus condition $\quad(2,9) \quad 25.82 \dagger$

$\begin{array}{lll}\text { New conjunction stimulus condition } & (2,9) & 12.26 \dagger\end{array}$

Main effect of counting versus no counting trials $(1,22) \quad 4.87 *$

across recognition conditions

Recognition performance between 3- and 15-sec delay

$\begin{array}{lll}\text { Experimental trials with counting } & (1,22) & <1.00 \\ \text { Control trials without counting } & (1,22)<1.00\end{array}$

${ }^{*} p<.05 . \quad \dagger p<.001$.

\section{Results}

The main results of Experiment 1 are shown in Figures 3 and 4 . The results of statistical tests are given in Table 1. Because there were no significant differences between the 3- and 15-sec delay conditions, these conditions were combined for further analysis. Figure 3 shows data from Sessions 2 through 5, in which the arithmetic distractor was used. The figure is divided into three panels, each of which represents results for the experimental condition described along the abscissa. Within each panel, the left-hand bar indicates the percentage of responses that fell into the first category, where the subject indicated that something new was present at test (empty bars). For the left panel, such responses were correct, and this fact is indicated by the letter $\mathrm{C}$ within the bar. The middle bars within each panel show the percentage of responses that indicated that the test item was identical to one of those in the inspection set (hatched bars). This was the correct response for the middle panel. The right-hand bars within each panel indicate a new conjunction of a form and color that had been seen in the inspection set; this is the correct response for the right panel (stippled bars). The bars within each panel sum to $100 \%$. The response range for the 4 subjects is shown near the top of each bar. The ranges indicated are, in every case, smaller than the obtained $95 \%$ confidence intervals.

About half of the responses overall fall into the first category, indicating that a new color, shape, or both had been detected. Such a response could occur because a subject remembered correctly that a test feature had not been present at inspection (left-hand bar of the left panel), or because he or she failed to remember one that had been present (left-hand bars of the middle and right panels). About half the responses of the experiment fall into this category despite the subjects' knowledge from the instructions that only a third of the test stimuli would contain novel features. 
The results in the left panel indicate that when something new was present at test, the subjects correctly indicated this about $80 \%$ of the time on average (the remaining responses being false alarms). This is considerably above the $50 \%$ to be expected by chance, given the distribution of the subjects' responses across categories. Salient features of the inspection set are remembered rather well, especially considering the powerful verbal distractor task that must be dealt with simultaneously.

The key results are shown in the middle and right panels. Recall that, in these two stimulus conditions, both the color and shape of the test item were present in the inspection set. The left-hand bars of each panel indicate that the subjects did rather well at discerning this, in the sense that only about $35 \%$ of their responses incorrectly indicate rejection of old features (misses). The remaining $65 \%$ of their responses (sum of the middle and right-hand bars of the middle and right panels) are at least partially correct in signifying that no new color or shape was added at test. However, the fact that there is no significant difference in the heights of these bars, either within or between panels, indicates a complete inability to remember whether or not the old features were conjoined at inspection.

By contrast, the very significant differences between bar heights displayed in the middle and right panels of Figure 4, representing results obtained when the distractor task was not used (the control condition), show that the conjunction of features (or lack of it) was rather well remembered. For example, subjects correctly indicated memory of old conjunctions (middle bar of middle panel) about four times as often as they did so incorrectly (righthand bar of middle panel). The corresponding ratio when new conjunctions were presented, shown by the relative heights of the middle and right-hand bars of the right panel, is more nearly two times, but still very significantly different from unity.

Aside from this vastly improved ability to remember conjunctions, performance was only a little better without than with the distractor task. In other words, the distractor task has a much more deleterious effect upon memory for conjunctions than for features.

The solution to the arithmetic problem was correct on $88.5 \%$ of the trials. For the 83 trials in which counting errors occurred, correct recognition was $55.4 \%$, not significantly better than the $47.9 \%$ correct recognition for trials on which the arithmetic problem was correctly solved.

Figure 5 shows recognition performance for sequential experimental sessions, with Session 5 being the control condition (recognition task performed without the counting-task distractor). Ranges providing the session performance of each subject individually are shown by the bars, with circles indicating the mean of the four scores. Although a practice effect is evident in the first four sessions, the large improvement in final session performance exceeds any improvement trend resulting from a practice effect.

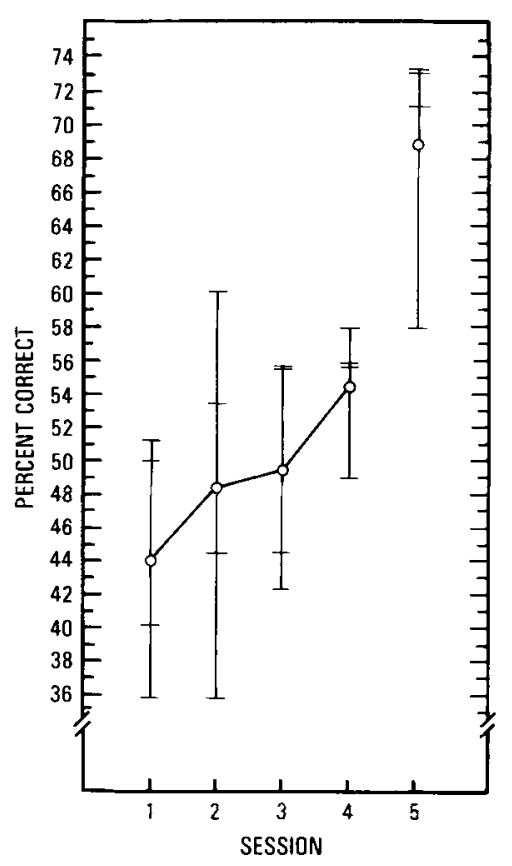

Figure 5. Practice effects in Experiment 1: Recognition performance as a function of session number. Error bars give the range of intersubject variability and intermediate scores.

\section{Discussion}

Although there are other possible interpretations, it seems to us to be likely that the kind of memory being tapped under the main conditions of the experiment is sensory in nature, largely unconscious, definitely not iconic, and possibly capable only of signaling whether or not something new is present at test.

The results reveal a difference between the recognition of structural features and conjunctional features when verbal resources for encoding, storage, and retrieval are consumed by the arithmetic task. When it comes to recognizing structural novelty (a new color or shape) in a test item, subjects who are almost as proficient with this distraction present as they are without it nevertheless fail to discriminate whether or not conjunctional shifts have occurred between inspection and test.

This dissociation of structural and conjunctional recognition performance is produced by the dependence of conjunctional recognition on a verbal component. Conjunction errors exceed feature recognition errors when verbal resources for initial tagging and subsequent combination of features across dimensions are made unavailable. This implies an essentially sensory memory for color and shape features, and a separate, verbally mediated memory for conjunctional features. A low-level feature-integration process, if it existed, would prohibit the conjunction errors so widespread in the results of this experiment. The combination or integration of features across dimensions apparently occurs after an initial stage of dimensional independence and, without the distractor task, it probably occurs during the inspection period. 
The independence of color and shape sensory memories in the face of our verbal distractor task would predict probability summation for one-third of the 240 trials in the new color or shape condition. This probability summation takes the form of increasing the chance of accurately identifying, as new, test stimuli presented on trials when both color and shape were new, relative to trials when only the color or the shape was new and the remaining dimension old. The subjects correctly identified $57.5 \%$ of the 80 new-shape trials as having something new at test, and identified $83.8 \%$ of the 80 new-color trials as being novel. Under the assumption of independence between color and shape memory processing, the predicted performance on the 80 trials having both new color and shape at test is $93.1 \%$ correct. The observed performance on these doubly new trials was $95.0 \%$ correct, which provides an additional strut in support of an independent sensory memory model.

The dependence of accurate conjunctional assignments upon verbal stages of featural coding and integration found here supports the findings of Treisman in her studies of illusory conjunctions. Our study does not address the issue of illusory conjunctions directly. The failure to recognize old conjunctions does not necessarily require the existence of a competing perceptual hypothesis. The forced-choice task produces guessing behavior apart from simple reports of perceptual content. Specifically, our task differs from those used by Treisman by adopting long exposure durations of inspection items in contrast to the tachistoscopic viewing conditions of her illusory conjunction work. Also, mechanisms of memory are involved in our three-alternative forced-choice task; these are absent in the reaction time studies of illusory conjunctions. Here, a sensory memory with a very slow decay rate works over intervals as long as $15 \mathrm{sec}$. Such memory may also mediate over much longer times, as in the experiments of Shepard (1967) and Haber (1970), whose subjects could accurately discriminate familiar from unfamiliar pictures of natural objects many hours after inspection.

The decision process leading to the choice of response could depend upon a simple feeling of novelty versus familiarity along a dimension. Recognition of structural oldness could depend upon a feeling of familiarity, or by default it could relate to the absence of a feeling of novelty. Neither process would require the explicit recall or identification of structural features at the time of the response. Therefore, processes leading to judgments of novelty could result from memory searches that do not return information about the target color or shape. Such a process would be mediated by independent nonverbal sensory memories that hold features of separate dimensions in storage and allow serial search procedures when the test item is presented.

The success of structural recognition when conjunctional recognition fails points to "separable dimensions" (Garner, 1977) for color and shape features. The current study serves to dissociate structural search from conjunctional search stages in serial processing while supporting independent searches along the dimensions of color and shape.

We are left with the conception of an information processing system that encodes and stores categorical dimensions in independent sensory memories which have a longer half-life than iconic stores. These memories may be sensory only in the sense that nonverbal imaginal, or possibly propositional, representations are involved. Verbal resources are necessary to integrate features across dimensions and to produce a higher level cognitive unit with inherent relational parameters. For dual-aspect stimuli, recognition is generated from serial searches within each dimensional store. Conjunctional recognition is generated from a search of working memory containing labels for featural pairs.

\section{EXPERIMENT 2}

This experiment differs from the previous one mainly in that, throughout each experimental session, subjects were told to pay attention only to shape or only to color and were rewarded accordingly. When changes occurred in conjunctions between inspection and test, these were irrelevant to payoff and could serve as aids or distractors only if conjunctions between colors and shapes had been formed and remembered. The arithmetic distractor was again used in an effort to eliminate encoding and memory of conjunctional relations.

\section{Method}

The stimuli were the same as those of the first experiment except that an additional animal (starfish) and color (gray) were added (see Figures 1 and 2). These served as possible perceptual aids or distactors along the irrelevant dimension. For example, if shape were the relevant dimension, a red camel at inspection might turn into a gray camel at test; or if color were the relevant dimension, a red camel at inspection might turn into a red starfish at test. The gray animal shapes were easily discriminated from the lighter gray background. There was no need to use gray starfish (such would be doubly irrelevant), so the total population of stimuli consisted of $(11 \times 11)-1=120$ different items.

The apparatus was the same as that used in the first experiment, except that there were only two response buttons, which were used to indicate whether or not the stimulus at test had the same shape (or, alternatively, the same color) as one of the five in the inspection set. The reward schedule was likewise the same, except that 104 rewards were given with a probability of .50 and that there were 10 experimental sessions that had to be completed before rewards could be collected.

The procedure within an experimental trial was the same as before, except for differences in instructions and reward contingencies and the availability of fewer response categories (now simply "same" or "different" along the one relevant dimension).

Eight subjects were tested using the same screening procedure as in the first experiment. No subject served in both experiments. Seven of the initial 8 subjects who began the experiment stayed the course. The other dropped out at the end of his second session, thereby forfeiting the rewards he had accumulated. His data were discarded and he was replaced.

Experimental design. The within-sessions variables were:

1. Delay between inspection and test. With the constraint that each delay would occur 10 times per session, delay intervals of 1 , 3,9 , and $27 \mathrm{sec}$ were varied randomly from trial to trial. 
2. Inspection stimuli. From a set of 10 items selected for use in a given session (a single condition was run per session), 5 were chosen randomly on each trial and positioned in an $\times$-like array according to the order of their selection. For some conditions (see below), the subset of 10 stimuli for single-session use was drawn randomly from a total set of 100 animals that were neither gray nor starfish, with the constraint that none of the 10 colors or shapes be repeated in the stimulus pool. Thus, each nongray color and each animal other than starfish was represented once in a session's stimulus pool and there was a unique association throughout a session (one that would differ between sessions) between animals and their colors when both were present at inspection or test. For some conditions, the inspection set, test item, or both were chosen from a stimulus pool consisting of either 10 starfish, each of a different nongray color, or 10 animal shapes, all of the same gray color.

3. Test stimuli. One item from the session stimulus pool of 10 was chosen randomly for presentation on each trial. The construction of the stimulus pool depended upon conditions that varied between sessions. These are summarized in Table 2 and are discussed below.

Sessions and Conditions. The subjects participated in 10 sessions. The first was an introduction, instruction, and practice session that took about $1 \mathrm{~h}$ to complete. Each of the next 8 sessions, which lasted about $90 \mathrm{~min}$, corresponded to one of the eight experimental conditions. The 10th session was used for a control condition in which the subjects were not required to perform mental arithmetic, and also for debriefing and discussion of the experiment.

For Condition 1, the subject was told that there would be a restricted set of 10 stimuli, to be used throughout that day's experimental session, both as inspection and test figures. These would vary in color and shape, but the color-shape relationships would be stable and unique between inspection and test. Although the subject was told to pay attention to color, his or her decision could equally well have been based on shape. Condition 2 was identical to Con-

Table 2 A

Between-Sessions Conditions of Experiment 2

\begin{tabular}{|c|c|c|c|}
\hline $\begin{array}{l}\text { Condition } \\
\text { Number }\end{array}$ & $\begin{array}{l}\text { Stimulus Aspects } \\
\text { at Inspection }\end{array}$ & $\begin{array}{c}\text { Stimulus Aspects } \\
\text { at Test }\end{array}$ & $\begin{array}{l}\text { Instruction } \\
\text { (Attend to:) }\end{array}$ \\
\hline 1 & Color and Shape & Color and Shape & Color \\
\hline 2 & Color and Shape & Color and Shape & Shape \\
\hline 3 & Color and Shape & Color & Color \\
\hline 4 & Color and Shape & Shape & Shape \\
\hline 5 & Color & Color and Shape & Color \\
\hline 6 & Shape & Color and Shape & Shape \\
\hline 7 & Color & Color & Color \\
\hline 8 & Shape & Shape & Shape \\
\hline 9* & Color and Shape & Color and Shape & Color \\
\hline
\end{tabular}

*Control.

Table 2B

Stimulus Items Used for Each Condition (Experiment 2)

\begin{tabular}{|c|c|c|c|}
\hline $\begin{array}{l}\text { Condition } \\
\text { Number }\end{array}$ & $\begin{array}{l}\text { Stimulus Items } \\
\text { at Inspection }\end{array}$ & $\begin{array}{c}\text { Stimulus Items } \\
\text { at Test }\end{array}$ & $\begin{array}{l}\text { Instruction } \\
\text { (Attend to:) }\end{array}$ \\
\hline $\begin{array}{l}1 \\
2 \\
3 \\
4 \\
5 \\
6 \\
7 \\
8 \\
9 *\end{array}$ & $\begin{array}{l}\text { Colored Animals } \\
\text { Colored Animals } \\
\text { Colored Animals } \\
\text { Colored Animals } \\
\text { Colored Starfish } \\
\text { Gray Animals } \\
\text { Colored Starfish } \\
\text { Gray Animals } \\
\text { Colored Animals }\end{array}$ & $\begin{array}{l}\text { Colored Animals } \\
\text { Colored Animals } \\
\text { Colored Starfish } \\
\text { Gray Animals } \\
\text { Colored Animals } \\
\text { Colored Animals } \\
\text { Colored Starfish } \\
\text { Gray Animals } \\
\text { Colored Animals }\end{array}$ & $\begin{array}{l}\text { Color } \\
\text { Shape } \\
\text { Color } \\
\text { Shape } \\
\text { Color } \\
\text { Shape } \\
\text { Color } \\
\text { Shape } \\
\text { Color }\end{array}$ \\
\hline
\end{tabular}

*Control. dition 1 except that the subject was told to pay attention to shape, and his or her decision could equally well have been based on color.

In Condition 3, inspection items were selected as for Condition 1, but the test item was always of the same starfish shape (shape-color relationships between inspection and test were therefore not constant). The test item defining Condition 4 could only be a gray animal; the inspection set was the same as in Conditions 1, 2, and 3.

In Condition 5, the inspection and test parameters of Condition' 3 were reversed. The inspection set contained only colored starfish, but the test item was chosen from a set containing colored animal forms. Conversely, the inspection set of Condition 6 contained only gray animals, each of which assumed a different color when seen at test.

For Condition 7, the inspection set and test item were drawn from a set of colored starfish (color was therefore the unique discriminating dimension). In Condition 8 , the inspection set and test item were drawn from a set of gray animals (shape was the unique discriminating dimension here).

The control condition differed from Condition 1 only in that the counting task was not used. In that session, 4 subjects were exposed to the tape recording, without being required to respond to it, and the other 4 were not so exposed (with no significant difference in the results). The 8 subjects of the experiment received the eight main experimental conditions in various orders, randomized and counterbalanced so that each condition would occur in a different session for each subject. All subjects received the control condition last.

Procedure for an experimental session. At the start of the session, the subject was informed whether color or shape was the aspect of the stimulus upon which a yes or no answer should be based. The experimenter then presented the 10 items comprising the stimulus pool for that session, in two sets of 5 , for $15 \mathrm{sec}$ each. To further acquaint the subject with the condition at hand, four unrewarded practice trials followed and the subject was reminded about the basis upon which his answers would be judged correct. Forty experimental trials were then run with a 5-min break midway.

As in the first experiment, eligibility for a reward depended upon the joint occurrence of a correct final sum on the arithmetic task and a correct response in indicating whether or not the form (or, alternately, the color) at test had been a member of the inspection set. The system of rewards and other feedback to subjects was unchanged from Experiment 1 . The span of 10 sessions lasted no more than 3 weeks for any observer and, with one exception, all sessions for a given observer occurred on separate days.

\section{Results}

Figure 6 summarizes the main results of Experiment 2. The right-hand ordinate shows the number of trials, for all subjects combined, on which the judgment of the test stimulus was correct-either that the test form or color had been a member of the five-member inspection set or that it was new. The left-hand ordinate shows an adjusted percent correct $\left(p^{*}\right)$ based on the formula $p^{*}=2 p-100$, where $p$ is the raw percentage correct. By this formula, $50 \%$ correct, which would be achieved on the average by a blindfolded subject, is adjusted to $0,100 \%$ correct is scored 100 , and a linear relation is assumed for intermediate values.

The error bars indicate $95 \%$ confidence intervals. The grand mean of $p^{*}$ for the eight main conditions of the experiment is $64 \%$, with individual means varying from $58 \%$ to $74 \%$ (a smaller range than the typical confidence interval). Analysis of variance confirms what the bar graphs suggest: there is no significant difference in per- 


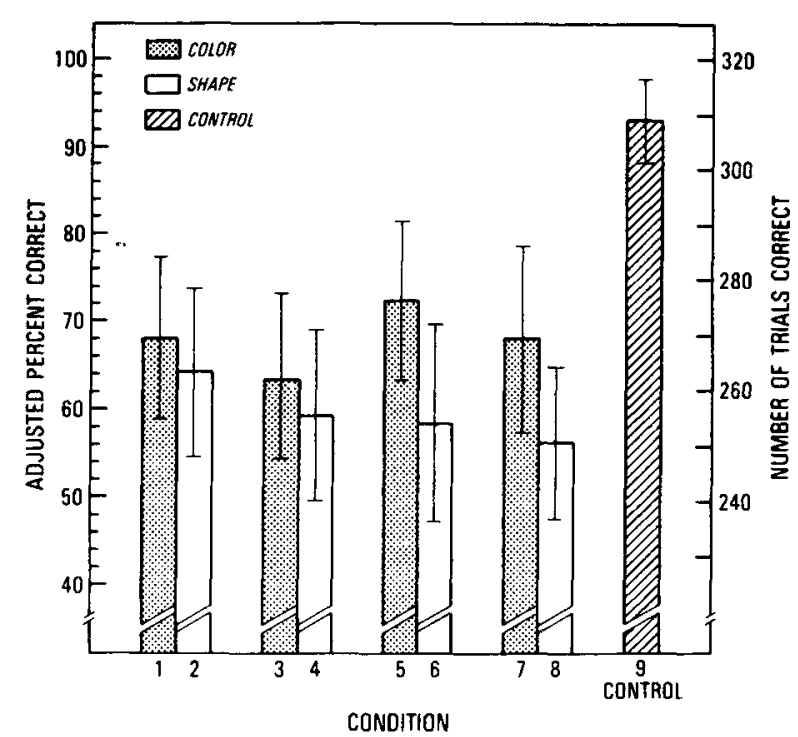

Figure 6. Main results from Experiment 2: Adjusted percent correct versus the eight experimental and single control conditions. Error bars give $95 \%$ confidence intervals.

formance associated with the eight experimental conditions $[F(7,42)=0.79, p<.60]$. The result for the control condition, in which the subjects were not obliged to solve the arithmetic problem, differs significantly from that of its Condition 1 counterpart $[F(1,6)=8.48$, $p<.03$ ].

Figure 7 shows the results plotted as a function of delay interval, with all other conditions combined. A significant difference between short (1 and $3 \mathrm{sec}$ ) and long ( 9 and $27 \mathrm{sec}$ ) delays is revealed by a specific comparison test $[F(1,6)=50.88, p<.001]$. There is no significant difference in the shape of this function regardless of whether the judgment was of color or of shape.

Transformed percent correct and $d^{\prime}$ scores were computed from the raw data. The results were consistent with analyses using raw percent correct as seen in the statistics above.

The solution to the arithmetic problem was correct on $\mathbf{9 1 . 5 \%}$ of the trials. For the 218 trials in which counting errors occurred, adjusted correct recognition was $53 \%$, significantly worse than the $65 \%$ correct recognition for trials on which the arithmetic problem was correctly solved.

Figure 8 shows recognition performance for sequential experimental sessions; Session 9 is the control condition. Mean performance is shown for the first eight sessions averaged over data from the eight experimental conditions (one per subject) run during each session, where the mean for Session 9 is the average from eight control conditions (one per subject). Error bars show 95\% confidence intervals. A practice effect is evident, with recognition performance for Sessions 1 through 4 differing significantly from that for Sessions 5 through $8[F(1,6)$ $=16.46, p<.007]$. The practice effect seems to be com- plete by Session 4: although Sessions 5 through 8 have largely overlapping confidence intervals with no significant change in mean performance, Session 9 (the control) shows no overlap.

\section{Discussion}

The demonstrated absence of facilitation or inhibition of recognition performance for color or shape in the presence of differences in the other dimension is consistent with the serial processing model suggested by Treis-

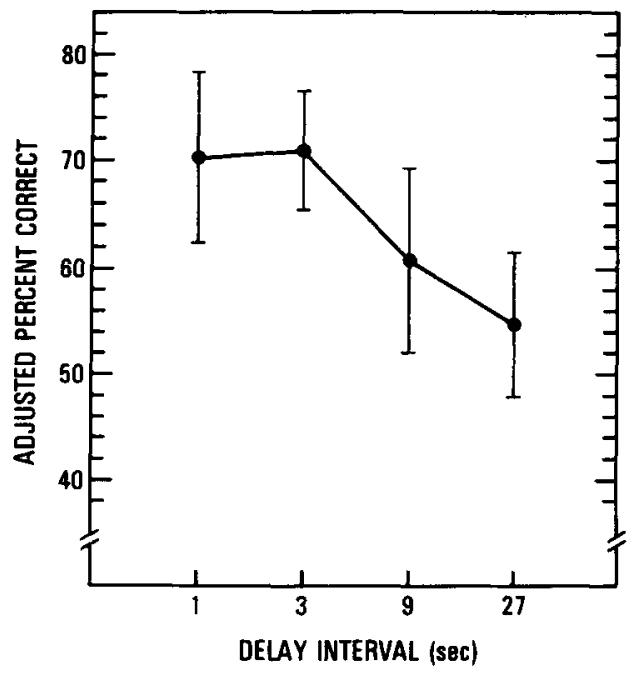

Figure 7. Results of Experiment 2: Adjusted percent correct as a function of delay interval with eight conditions and eight subjects combined. Error bars give $\mathbf{9 5 \%}$ confidence intervals.

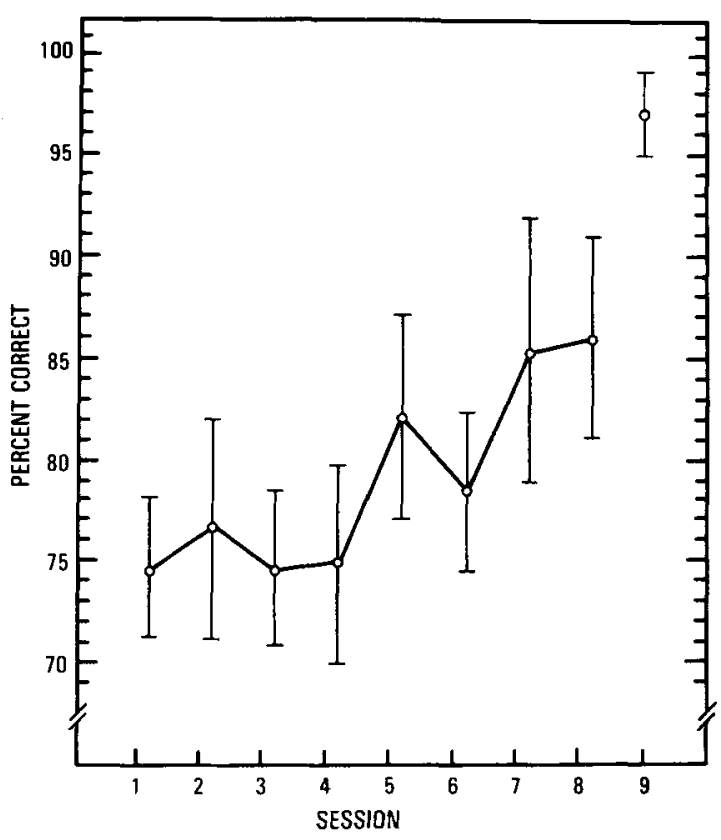

Figure 8. Practice effects in Experiment 2: Recognition performance as a function of session number. Error bars give $95 \%$ confdence intervals. 
man (1977) and with the results and interpretation of Experiment 1 . Therefore, the lack of significant differences among the scores for the eight main conditions in the experiment does not constitute a negative result in the usual inconclusive sense. The data are sufficiently precise for any important differences in performance that might be related to differences in the experimental conditions to have been evidenced in this experiment. Obviously, we cannot claim that statistically significant differences might not emerge if the study were run with more subjects or for a greater number of trials. But, there is no reason to believe that, were the experiment to be replicated indefinitely, the differences between the means would become significantly larger than the ones we have observed. With this proviso, important conclusions can be drawn from these negative results.

Most importantly, when forms and colors differ categorically and naming is inhibited, the results of this experiment add to those of Experiment 1 in showing an astonishing degree of independence between whatever mechanisms of memory for color and shape allow them to be recognized as long as half a minute later. For subjects instructed to pay attention to only one dimension of the stimuli, the presence of a second dimension, whether or not it is correlated with the first, neither assists nor interferes with perception and memory associated with the primary task. The failure even to find evidence of probability summation suggests that, for Conditions 1 and 2 , in which both cues were available, the subjects concentrated, as instructed, on only one of them. When colors were tested, then colors were all that mattered (Condition 1). When forms were tested, then forms were all that mattered (Condition 2). When colors alone were presented and tested (Condition 7), there was no penalty for not having the redundant form cues available. When forms alone were presented and tested (Condition 8), there was no penalty for not having the redundant color cue available. When both dimensions were available but one of them was not useful and could have served as a distractor, either because it was not present at inspection or because it was eliminated at test (Conditions 3 through 6 ), no loss of performance resulted.

\section{GENERAL DISCUSSION}

\section{Methodology}

Effectiveness of the arithmetic task. Without the competition of the arithmetic task, the tendency to encode a representation of the inspection set by using names is both natural and irresistible. In Experiment 2, the subject learned about the distribution of stimuli for that day during the practice period at the start of each experimental session. The data of the control condition show that, if the use of various strategies involving categorization by names is not prevented, a practiced subject can almost perfectly retain information about which 5-member subset of the 10 possible stimuli had appeared in the inspection set.
The arithmetic task, which a typical subject could almost always perform correctly in preliminary tests if confronted with that task alone, was chosen because it was deemed to be sufficiently demanding for even a small reduction of the 2-sec interinteger interval to lead to a significant reduction in accuracy. The task was designed to occupy a significant fraction of the subject's cognitive resources, including especially whatever hypothetical semantic machinery might be required to organize memory for forms and colors through the naming of them. At first, judging from our own impressions and those of the subjects' verbal responses, we did not believe we were entirely successful. From listening to recordings that were made of the subjects' verbal output, we detected a tendency for the rhythm of their answers to alter when the period for solving the problem overlapped that of the inspection period. On the basis of this observation and the subjects' introspections, we felt that they might momentarily have switched attention from the arithmetic task to the colors and forms-probably using names to some extent-meanwhile trusting their echoic auditory memory to bridge the resulting temporal gap and thereby permit resumption of the mental arithmetic with an acceptable risk of error. Nevertheless, the mental arithmetic took its toll on the perceptual task. In Experiment 1, it succeeded in destroying memory for conjunctions between color and form completely. In Experiment 2, by the eighth regular session, visual performance with the arithmetic distractor task was still much worse than it was in the control condition that immediately followed. In the context provided by the results of the first experiment, it seems reasonable to conclude that, in the control condition of the second experiment, in which the arithmetic task was not employed, the subjects were able to verbally tag and use the stable conjoint relationship between the shape and color with which they were presented.

Rewards. The fact that we used a system of rewards, which is a rather uncommon procedure for experiments in perception and cognition, allows us to extend our conclusions concerning the extent to which we may have succeeded in suppressing the use of names. The accumulated rewards were very substantial, and their effectiveness was strengthened on every trial by the possibility of a dollar reward for a single correct response (essentially a concurrent variable ratio schedule). There can be no doubt that the subjects' strategy-one that we did nothing to discourage-was to earn money by "beating the system" in the most efficient way possible. They knew that the correct solution to the arithmetic task was absolutely required for a reward, and that there was only one chance in nine to achieve this by guessing. On the other hand, they also knew that there was a much better chance of being right on the perceptual task, even if they saw nothing ( $p=.33$ in Experiment $1, p=.50$ in Experiment 2). The optimal strategy - the one we hoped subjects would adopt-was to concentrate on the arithmetic task while looking in a relatively relaxed and passive way at the visual display, relying on some kind of nonverbal and 
largely unconscious memory during a delay period of unpredictable length, to achieve a reasonably high proportion of correct recognition responses.

By using rewards, we minimized individual differences and also achieved an unusual precision of results and a very high level of performance for so demanding a task. For example, while correctly solving the arithmetic problem, subjects of Experiment 2 were correct about $50 \%$ of the time (after adjustment for chance) even after the 27-sec delay. Good as this performance was, it should be compared with nearly perfect performance for the same delay without the competing arithmetic task. In the first experiment, very clear results were obtained and only four subjects were needed. In both experiments, all subjects behaved in a consistent manner. Recall that in Experiment 1 the intersubject ranges in performance were smaller than the associated $95 \%$ confidence intervals. Therefore, the pattern of data and conclusions related thereto apply to subjects individually as well as collectively. We conclude that the use of rewards and the distactor task largely accomplished the aims of controlling behavior in desired ways.

\section{Major Conclusions}

We conclude that when objects differ categorically, there is an aspect of visual encoding and memory, neither semantic nor imaginal, that operates independently and equally well for form and color and helps to determine the likelihood of initial registration and later memory of those objects. When the use of names is inhibited, memory for features is only slightly affected, whereas memory for conjunctions is abolished.

Problems of encoding. Even had we succeeded in suppressing naming completely-which we cannot claimwhat remains would not represent a pure study of memory. Uchikawa and Ikeda (1981) have shown that there is only a modest loss of discrimination for very small color differences after a 5-sec delay, as compared with simultaneous presentation of stimulus pairs. From the fact that performance in Experiment 2 with a mere 1-sec delay was far from perfect we conclude that some of the stimuli in the inspection set were typically not encoded in any form. Perhaps most of the performance loss for the two shorter delays, relative to the control condition, should not be regarded as a memory loss at all, but rather as a failure of the stimuli to register, caused by the diversion of attention to the competing arithmetic task. If so, memory of form and color during longer intervals for those stimuli that actually registered may be considerably better than the raw data would suggest. This situation is similar to the inability of many people to remember the names of those to whom they are introduced because of a failure to pay attention to the names in the first place. Additionally, stimulus attributes can be separated (and recombined) during early perceptual processing. It is true that the constraints of initial registration and categorization will delimit subsequent information processing, but in a complementary manner later processing must support initial attribute transformations to allow the construction or computation of a useful cognitive representation. Here, the specific stage(s) of information processing that is affected by the arithmetic resource competitor is not identified by the presented methodology. Given previous evidence that attentional factors regulate early perception (Treisman, 1977), we accept the possibility that there is a registration focus to our demonstration of a dissociation of color and form in "'memory." Because memory begins with the encoding of information from the environment and cannot occur without it, a clear separation between mechanisms of encoding and memory is inherently difficult to make. The word "memory" has been used in this paper to embrace both aspects of the problem.

In the absence of "verbal glue," a remarkable separation of memory for form and color occurs which most likely relates to their separation during the encoding process; the use of names (or some process highly correlated with it) also allows color-form connections to be encoded and remembered.

Some suggestions for further research. It is possible that the memory trace fades more slowly for conjoined entities (of apparent verbal construction) than for features. A comparison of the results of the 3- and 15-sec control conditions of Experiment 1 fails to confirm the difference to be expected if this hypothesis is correct, but the small quantity of data in these cells is insufficient to permit firm conclusions, and the specific times chosen may not be significantly different.

Additional research could test the paradigm of Experiment 1 with animals and aphasic humans. This would help to clarify whether the "glue" that seems to hold forms and colors together is necessarily verbal in the sense of human speech production. Some other representational process, associated with naming but not identical with it, could provide the basis of memory for conjunctions of dual-aspect stimuli under the conditions of our experiments.

\section{REFERENCES}

BARTLESON, C. J. (1960). Memory colors of familiar objects. Journal of the Optical Society of America, 50, 73-77.

BERLIN, B., \& KAY, P. (1969). Basic color terms, their universality and evolution. Berkeley: University of California Press.

GARNER, W. (1977). The effect of absolute size on the separability of the dimensions of size and brightness. Bulletin of the Psychonomic Society, 9, 380-382.

Haber, R. (1970). How we remember what we see. Scientific American, 222, 104-115.

HeIDER, E., \& OLIVER, D. (1972). The structure of the color space in naming and memory of two languages. Cognitive Psychology, 3, 337-354.

Herring, B., \& Bryden, M. (1970). Memory colour effects as a function of viewing time. Canadian Journal of Psychology, 24, 127-131.

PAIvio, A. (1969). Mental imagery in associative learning and memory. Psychological Review, 76, 241-263.

RATLIF, F. (1976). On the psychophysiological bases of universal color terms. Proceedings of the American Philosophical Society, 120, 311-330.

SANTA, J. (1977). Spatial transformations of words and pictures. Jour- 
nal of Experimental Psychology: Human Leaming \& Memory, 3, 418-427.

Schiano, D. J., \& WatKINs, M. J. (1981). Speech-like coding of pictures in short-term memory. Memory \& Cognition, 9, 110-114.

SHEPARD, R. (1967). Recognition memory for words, sentences, and pictures. Journal of Verbal Leaming \& Verbal Behavior, 6, 156-163.

Treisman, A. (1977). Focused attention in the perception and retrieval of multidimensional stimuli. Perception \& Psychophysics, 22, 1-11.

Treisman, A., \& Schmidt, H. (1982). Illusory conjunctions in the perception of objects. Cognitive Psychology, 14, 107-141.

UCHIKAWA, K., \& IKEDA, M. (1981). Temporal deterioration of wavelength discrimination with successive comparison method. Vision Research, 21, 591-595.

\section{NOTE}

1. In the first experiment, nine of the colors were named with perfect consistency. One subject used the name peach to refer to what others called purple. In the second experiment, seven of the colors were named with perfect consistency. One subject used the name chocolate to refer to what others called brown. One subject used brown instead of black. The sample called purple by six subjects was identified as maroon or magenta by the others. What was intended to be gray (23\% darker than the background) was variously called gray (3), beige (2), tan (2), and taupe (1). For the animals of the first experiment, there was perfect agreement in the naming of 7 out of 10 . One subject accounted for all of the alternate responses: bird for ostrich, horse for camel, and shark for whale. For the subjects of the second experiment, there was perfect agreement in the naming of all but two of the animals. What seven subjects called starfish was a seastar for the eighth; one subject called what was an ostrich for the others a peacock.

(Manuscript received October 28, 1985; revision accepted for publication March $12,1986$. 